\title{
Overview and examples of instructional modules
}

\author{
PHILIP F. SPELT \\ Wabash College, Crawfordsville, Indiana
}

\begin{abstract}
The fundamental instructional design concepts underlying the systematic development of new computer-based courseware are defined and illustrated. The advantages of the electronic chalkboard and electronic studyguide are discussed.
\end{abstract}

\section{OVERVIEW OF PRESENT MODULES}

One of the primary goals of the task force was to design an initial set of modules that would provide as broad a coverage of "typical" introductory psychology topics as possible and still allow for meaningful vertical integration into subsequent intermediate- and advancedlevel undergraduate courses (Castellan, 1984). Thus, some of the topics covered in a survey text such as Gleitman's (1981) would not be suitable, since they are unlikely to be represented in an advanced course. These constraints, coupled with the particular interests and expertise of the participants, shaped the choice of the initial five module topics: the nature of psychology, methodology, sensation and perception, conditioning and learning, and human memory. Not surprisingly, the first two of these modules are more process oriented, and the remaining three are more content oriented.

Each module is designed to present a well-defined set of topics by a method that combines the acquisition of facts and concepts with some graphic demonstrations and the opportunity to "experiment" with the materials and processes of the unit as they are presented by the microcomputer. The acquisition of the factual material is accomplished with traditional computer-assisted instruction (CAI) techniques (e.g., drill and practice, tutorial units) blended with sections that allow the student to pursue avenues of interest by asking the machine for more information on specific topics. The graphic demonstrations are largely included in the "electronic chalkboard" concept reported in some detail by Spivey (1984). There are also other items that make use of computer graphics as part of the "electronic study guide" aspects, as will be illustrated in the discussion of a particular module.

\section{AN EXAMPLE OF APPLICATION}

The conditioning and learning module is a good vehicle for illustrating the meaning of the various concepts that coalesce to make these modules very different from traditional CAI. This module is designed to

The author's mailing address is: Department of Psychology, Wabash College, Crawfordsville, IN 47933. mesh with the traditional conditioning and learning chapters of typical survey-type introductory texts, such as those of Atkinson, Atkinson, and Hilgard (1983), Gleitman (1981), Zimbardo (1979), and others. The areas covered are classical and instrumental conditioning, from both the appetitive and aversive perspectives, and a subsequent blending of the two types into an integrated model of incentive learning that serves as a springboard to a more cognitive view of conditioning and to the human memory module.

The electronic chalkboard, which enables the instructor to select some elements of the module for classroom viewing, is intended to provide demonstrations that the student can then pursue further outside class, during individual study time for the course. In the case of classical conditioning, the class demonstration consists of using the keyboard to deliver "stimuli" to the subject, a dog undergoing Pavlovian salivary conditioning. In class, the instructor presents the concepts of UCS $\rightarrow$ UCR, CS, and CR, using the microcomputer in the following way.

As the chalkboard presentation begins, a figure of a dog with the proper fistula and UCS delivery tube is displayed on the screen. The instructor then presses the [U] key, which results in the appearance of the symbols "UCS $\rightarrow$ UCR" on the screen and the delivery of some acetic acid down the tube in the graphic, followed by some drops of saliva in the graduated beaker. The nature of the reflex relationship between USC and UCR can then be discussed. Pressing the [C] key results in the placing of a "CS" on the screen, to the left of the symbols for the UCS-UCR, coupled with a beep from the machine to represent a tone (see Figure 1). After a discussion of CS characteristics and of the CS-UCS pairing procedure in classical conditioning, the students are prepared to go home and work with the module, exploring the CS-UCS interval. They do this by first specifying the type of conditioning (simultaneous, delayed, trace, or backward) and then specifying the interstimulus interval, if appropriate. "Conditioning" is then simulated, and an acquisition curve is presented on the screen. Thus, in this case the electronic chalkboard leads directly into the electronic study guide concept.

"Electronic study guide" refers to the fact that the student can use part of the electronic chalkboard unit as 

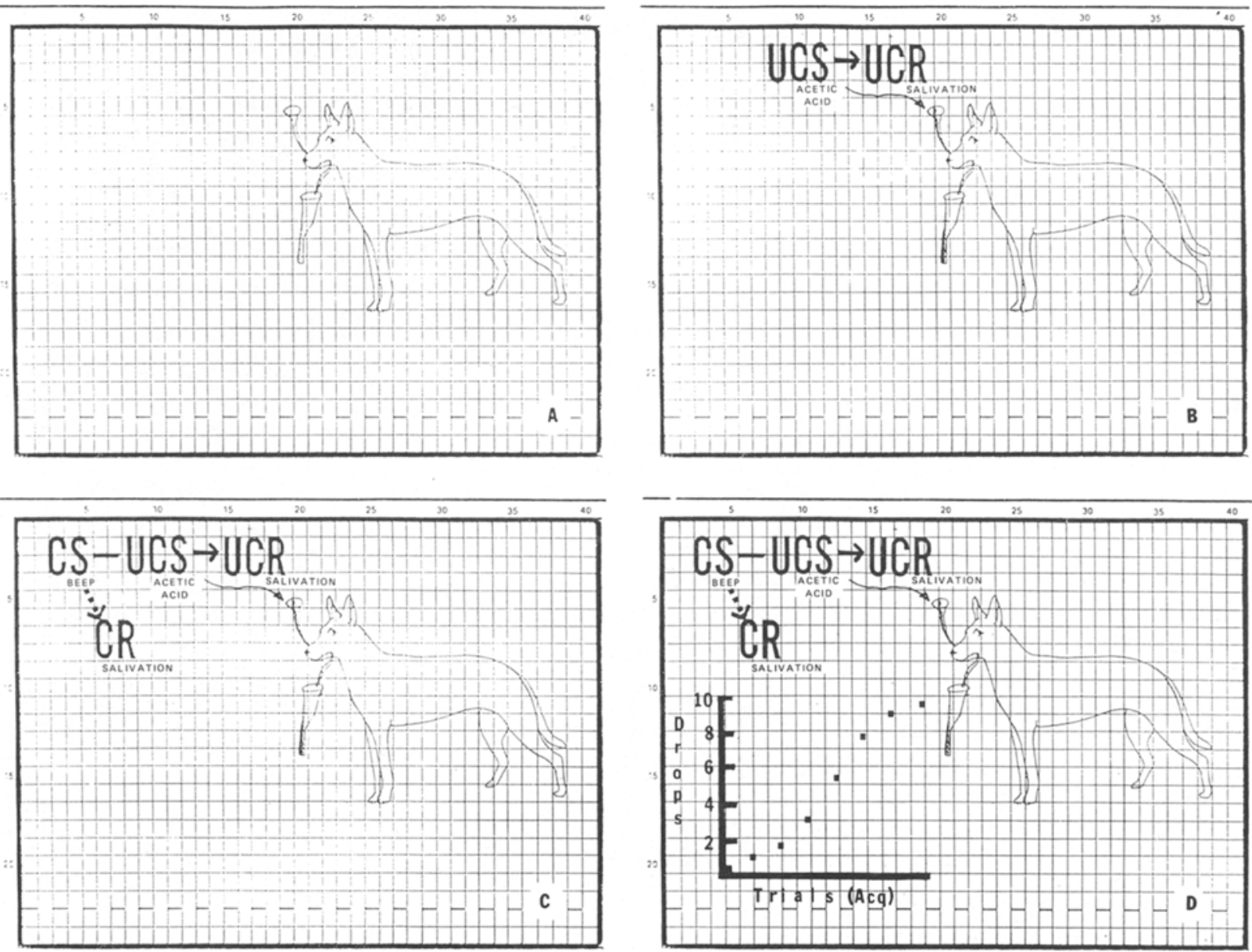

Figure 1. Storyboard diagram of the classical conditioning graphic for the electronic chalkboard in the conditioning and learning module. Panels A through D show the progressive use of text and graphics ports to display successive elements of the chalkboard.

the basis for study and exploration outside class. The integration of the features of study guide and chalkboard allows the student to probe the contents of the unit that was initially presented in class. In the conditioning and learning module, four types of conditioning are available for the student to explore on his/her own, along with the interval between stimuli:

(1) Simultaneous conditioning-This type of conditioning, which presents the CS and the UCS at exactly the same time, produces relatively little acquisition, a result that is consistent with much of the literature.

(2) Delayed conditioning-with this standard conditioning procedure, the CS is presented, followed very briefly by the UCS (while the CS is still on). On the basis of the CS-UCS interval that the student selects, acquisition proceeds relatively slowly or quickly, depending on how close the interval is to $0.5 \mathrm{sec}$. The student is encouraged to explore several temporal relationships between the CS and the UCS.

(3) Trace conditioning-Trace conditioning is similar to delayed conditioning, except that the CS goes off before the UCS comes on. If the student selects this method, acquisition proceeds relatively slowly, and the longer the delay, the weaker the conditioning effect.

(4) Backward conditioning-This procedure reverses the order of the two stimuli. As is consistent with the American literature, no conditioning occurs with the UCS $\rightarrow$ CS sequencing.

With this set of study guide demonstrations, the student can actively explore the CS-UCS interval at his/her own pace, and can build on the classroom demonstration based on the electronic chalkboard. By this process, the student is led to the general conclusion that delayed conditioning with a CS $\rightarrow$ UCS interval of about $0.5 \mathrm{sec}$ produces the strongest effects, a finding that can be reinforced in later class discussion.

Probing of the computer by the student, rather than having the machine direct all of the learning process, is a key innovation of these modules. The intention is to reverse the usual roles of teaching machine and learner, allowing the student to explore avenues of interest to him/her at that time. This student-directed learn- 
ing is facilitated by easy return to the main menu-the overall "map" of the module-from any point in the module. It is facilitated also by the fact that the student may choose among the units presented in the menu, subject only to the limitations imposed by required background knowledge. The opportunity for student probing is represented in the classical conditioning unit presented above by the variable sequencing of the types of conditioning coupled with a reminder that the student should explore several temporal relationships between CS and UCS.

Throughout, the modules demonstrate concepts, rather than merely telling the student about the phenomena and ideas. Again, this is illustrated by the classical conditioning unit described above, and this characteristic is incorporated at all levels and in all units of the modules. For instance, the concept of shaping in instrumental conditioning is illustrated through the use of a simulation adapted from a program that allows the student actually to "guide" a mouse into a goal box by the use of reward, punishment, or both. This simulation permits the student to explore the contingency relationship between an instrumental response (jumping toward or away from the goal) and the delivery of the reward or punishment. Vicarious trial and error (VTE) is actually demonstrated in a simulation of T-maze learning in rodents (RATS program), which shows a subject running to the choicepoint and looking right and left before choosing a goal.

"Horizontal integration" refers to the similarities among the various modules, and has two aspects: between and within modules (Castellan, 1984). Betweenmodule horizontal integration is reflected most obviously in the similarities in mechanics by which the student progresses through the various modules. They are all menu driven, and the mechanics of selecting various entries in a menu are the same throughout. In addition, where possible and appropriate, the technical terminology used in the various modules is standardized. Finally, the presentation and labeling of graphics are standardized across modules. With this sort of crossmodule standardization, the student should feel "at home" in any of the modules.
Horizontal integration within a module is easier to obtain, at a superficial level, since each module is authored by a single individual. However, the more important horizontal integration-integration of concepts- is difficult even with only a single author. In the conditioning and learning module, the task is relatively straightforward both because the terminology has been standardized and because the introduction of a classically conditioned incentive-motivational concept into instrumental conditioning serves both to integrate the two conditioning paradigms and to provide a springboard into an information-processing approach to learning and memory. The latter approach provides the necessary "hooks" to link with the human memory module, as well as with a future module on human cognitive processes in general.

"Vertical integration" refers to the fact that concepts, demonstrations, and simulations introduced at lower levels are continued and expanded at more advanced levels, thereby allowing students to pursue concepts at more complex levels as either their curiosity or their enrollment in higher level courses demands (Castellan, 1984). Thus, the conditioning and learning module, which presents the fundamentals of classical, freeoperant, and (discrete-trial) instrumental conditioning at the introductory level, permits a more complex and thorough investigation of these topics during the intermediate-level learning course.

Beginning at the intermediate level, and continuing in the advanced level, some important changes are introduced. More complex data-generating models of the EXPER SIM type (Main \& Head, 1971; Spelt \& Schafer, 1976) are introduced, and the "Sesame Street" graphics of the beginning units are eliminated. The purpose of this shift of strategy is to shape the student into a more rigorous empirical approach to the study of psychological phenomena. For example, the simulation of shaping is expanded into a simulation of free-operant conditioning that permits exploration of the various schedules of reinforcement. The VTE simulation of T-maze learning is likewise expanded into the TMAZE simulation (Spelt \& Warden, 1975) from the EXPER SIM series of models. TMAZE permits the investigation of

Table 1

Pattern of Units in the Conditioning and Learning Module, Showing the Development of Concepts and Complexity Through the Three Levels of Instruction

\begin{tabular}{|c|c|c|c|}
\hline \multirow[b]{2}{*}{ Level } & \multicolumn{3}{|c|}{ Concepts to be Taught } \\
\hline & Classical Conditioning & Free-Operant Shaping & Discrete-Trial Reinforcement, VTE \\
\hline Introductory & $\begin{array}{l}\text { Acquisition } \\
\text { Extinction } \\
\text { CS-UCS interval }\end{array}$ & $\begin{array}{l}\text { Acquisition/Extinction } \\
\text { Shaping with reinforcement } \\
\text { and with punishment }\end{array}$ & $\begin{array}{l}\text { Discrimination learning } \\
\text { Reinforcement } \\
\text { VTE }\end{array}$ \\
\hline Intermediate & & $\begin{array}{l}\text { Schedules of reinforcement } \\
\text { (expanded) }\end{array}$ & $\begin{array}{l}\text { Problem difficulty } \\
\text { Training procedure }\end{array}$ \\
\hline Advanced & Pavlovian injection & Discrimination learning & $\begin{array}{l}\text { SR phenomenon } \\
\text { Shock intensity and } \\
\text { duration }\end{array}$ \\
\hline
\end{tabular}


such variables as training procedure (whether or not the animal is allowed to correct an error) and problem difficulty (how similar the discriminative stimuli are) at the intermediate level, and a thorough investigation of the shock-right facilitation (Spelt \& Fowler, 1969) phenomenon at the advanced level (see Table 1).

Properly guided, students at the intermediate and advanced levels will learn both subject-area content and methodology by exploring these units. There is opportunity, if not necessity, for the student to work individually with the instructor or a teaching assistant to develop the skills involved in casting research questions in testable form and in seeking the data from which to derive answers to those questions.

\section{SUMMARY}

The modules included in this series are intended to serve a new function in the teaching-learning process. Through the electronic chalkboard, they give the teacher a method of demonstrating concepts and phenomena in the classroom, and at the same time allow the students to take a significant part of the class activity home to explore in his/her own style. The electronic study guide home activity allows self-pacing and scheduling of study activities, and also provides the opportunity for the student to explore some personally attractive avenues that may go beyond the limits of the particular assignment. Properly integrated into the course, these exercises will be reinforced by class discussion, and will provide a much more active way of learning the material. Because of the horizontal and vertical integration designed into the modules, they are usable throughout the undergraduate career, and the student therefore should come away with a more integrated view of the various areas of psychology. Concepts introduced at the beginning level are more explicitly and clearly reinforced and expanded at the advanced levels. If the traditional printed study guides have been successful, then the electronic form, coming as it does at the height of the personal computer revolution, should be even more so, since the flexibility of the microcomputer allows and even encourages activities that the printed form cannot.

\section{REFERENCES}

Atkinson, R. L., Atkinson, R. C., \& Hilgard, E. R. (1983). Introduction to psychology. New York: Harcourt Brace Jovanovich.

Castellan, N. J., Ja. (1984). A model for courseware development in psychology. Behavior Research Methods, Instruments, \& Computers, 16, 165-167.

Gleitman, H. (1981). Psychology. New York: Norton.

Main, D. B., \& Head, S. (1971). Computer simulations in the elementary psychology laboratory. Proceedings of the Conference on Computers in the Undergraduate Curricula, Dartmouth.

Spelt, P. F., \& Fowler, H. (1969). Shock-right discrimination training: Effect of correction training with an enforced delay following an incorrect choice. Journal of Experimental Psychology, 79, 504-508.

Spelt, P. F., \& Schafer, S. R. (1976). The use and evaluation of EXPER SIM: What does it teach and how should it be evaluated? Proceedings of the Seventh Conference on Computers in the Undergraduate Curricula, Binghamton, NY.

SPELT, P. F., \& WARDEN, J. A. (1975). WRIST-Wabash Research Investigation simulation teacher, student's and instructor's guide. Maynard, MA: DECUS Program Library, Digital Equipment Corporation.

SpiveY, J. E., \& Jackson-Smith, P. (1984). The electronic chalkboard. Behavior Research Methods, Instruments, \& Computers, 16, 172-175.

Zimbardo, P. G. (1979). Psychology and life. Glenview, IL: Scott, Foresman. 